Check for updates

Cite this: RSC Adv., 2017, 7, 25253

Received 24th January 2017

Accepted 5th May 2017

DOI: 10.1039/c7ra01076a

rsc.li/rsc-advances

\section{Pyrolysis products and thermal degradation mechanism of intrinsically flame-retardant carrageenan fiber}

\author{
Zhixin Xue, iD *ab Weiwei Zhang, ${ }^{\text {ab }}$ Miao Yan, ${ }^{\text {ab }}$ Jingjing Liu, ${ }^{\text {ab }}$ Bingbing Wang ${ }^{\text {bc }}$ \\ and Yanzhi Xia*b
}

Carrageenan fiber (CAF) was prepared by a wet spinning method to develop an excellent flame-retardant material. In order to investigate the flame-retardant mechanism of CAF, a series of tests were carried out. The values of limiting oxygen index (LOI), total heat release (THR), and total smoke release (TSR) of CAF reached up to $52,2.9 \mathrm{MJ} \mathrm{m}^{-2}$, and $2.5 \mathrm{~m}^{2} \mathrm{~m}^{-2}$, respectively, while there was no Time To Ignition (TTI) in cone calorimeter (CONE). These results indicate that CAF exhibits superior flame-retardant performance than other alginate fibers. Thermogravimetry, differential scanning calorimetry, Fourier transform infrared spectroscopy (TG-DSC-FTIR) and pyrolysis-gas chromatography-mass spectrometry (Py-GC-MS) tests were performed in order to explore the pyrolysis mechanism. The results show that CAF absorbed more heat and produced more carbon residue than calcium alginate fiber (ALF) and agar fiber (AGF) in the process of thermal degradation. In addition, in the current work, we have explored the possible flame-retardant mechanisms which can be attributed to sulfonyl free radical (SFR), carbon residue, barium sulfate, water vapor, and carbon dioxide.

\section{Introduction}

The increasing demand for novel functional materials and environmental protection materials has constantly encouraged people to engage in the research for new products. A few studies reported in the literature have found that carrageenan fiber (CAF) is a renewable and non-polluting natural seaweed product in contrast with the conventional petroleum-based fibrous materials. ${ }^{1}$ Hence, CAF shows an extensive potential to be applied in textile fabrics, such as fire-protection suits, bedclothes, curtains and carpets.

Carrageenans belong to the biopolymer family and have been widely used as food additives for their beneficial health properties. The flame retardancy is another innovative feature of carrageenans, which is well worth studying in order to produce flame-resistant materials. Carrageenan is a sulphated polysaccharide and has a random coil conformation in aqueous solutions when the temperature of the solution is higher than the gelation temperature. ${ }^{2,3}$ Carrageenans are extracted from red algae, and the most important members are kappa $(\kappa)$, iota ( $)$, and lambda $(\lambda) \cdot{ }^{4-6}$ Additionally, they carry a different

${ }^{a}$ College of Chemistry and Chemical Engineering, Qingdao University, Qingdao 266071, China

${ }^{b}$ Research Institute of Marine Fiber Novel Materials, Qingdao University, Qingdao 266071, China.E-mail: xyz@qdu.edu.cn

'Yantai Tayho Advanced Materials Group Co., Ltd, No. 9 Heilongjiang Rd., Yantai EDTA 264000, China number of sulfate groups. The structures of the macromolecules are composed of linear polysaccharides of alternating 3linked $\beta$-D-galactopyranosyl residues and 4-linked $\alpha$-D-galactopyranosyl or 3,6-anhydro-galactopyranosyl residues, while the sulfate esters are introduced in different positions. ${ }^{7,8}$ Due to the polyanionic characteristic of sulfate bases, carrageenans exhibit many biological activities, such as acting as an anticoagulant or anti-viral. ${ }^{9-14}$ Also, sulfate bases can play a key role in flame retardancy when carrageenans are spun into threads. ${ }^{15,16}$

Recently, most of textiles have been dealt with phosphorusbased flame retardant, halogen-based flame retardant, inorganic flame retardant, and nitrogen-containing flame retardant to reduce the flammability. ${ }^{\mathbf{1 7 - 2 4}}$ The applications of those flame retardants have been the most efficient ways of reducing the economic loss and ensuring personal security when the fire accident happened suddenly. However, once burned, some of the flame retardants produce corrosivity, carcinogen, and poisonous gas, ${ }^{18}$ which violate the principle of environmental protection. Nevertheless, CAF possesses the function of flame retardancy and pro-environment, which will be attached importance to its practical application. This double-win behavior has attracted much attention in recent time and continues to attract significant interest in the field of flame retarded applications, especially fire resistant fiber.

In recent years, Professor Xia and coworkers have explored the flame-retardant mechanism of alginate fibers and cellulose fibers. ${ }^{25-27}$ The group has already shown that divalent metal ions 
play an important role in the flame retardancy and thermal stability of alginate fibers. Calcium alginate fiber (ALF), barium alginate fiber and zinc alginate fiber have good fire-retardant properties without addition of other conventional flame retardants. ${ }^{28-30}$ The aforementioned results proved that the addition of divalent metal ions effectively improved the flame-retardant properties of the fabrics. Coincidently, coagulation bath, in the presence of barium ions, was applied to wet spinning to obtain the CAF with divalent metal ions (barium ions) into its molecular structure.

In the current study, CAF produced through wet spinning method, is demonstrated as a novel and an extremely efficient flame retardant material. ${ }^{31}$ The discussed method proved to be exceptionally advantageous when we reasonably utilize the viscoelasticity and gelation of carrageenan solution. ${ }^{32-37}$ In regards to the flame-retardant properties of the material, molecules with higher content of sulfate ester can deliver more active flame retardant atoms. Agar is another polysaccharide extracted from red algae, whose molecular structure is similar to the carrageenan, except lower sulfate content.

In this study, the flame-retardant properties of agar fiber (AGF) and ALF were investigated for the purpose of comparisons with CAF. Meanwhile, the flame-retardant mechanism of CAF and its pyrolysis mechanism are also investigated in the current work.

\section{Experimental section}

\subsection{Materials}

Kappa carrageenans $\left(\kappa^{-}\right)$were purchased from Shishi Universal Joan Glue Industrial Co., Ltd (Quanzhou, China). Agar (biological reagent) was obtained from Lanji Technology Development Co., Ltd (Shanghai, China). Barium chloride, dimethyl sulfoxide, ethanol, and sodium hydroxide were purchased from Sinopharm Chemical Reagent Co., Ltd (Shanghai, China), and these reagents were all analytical grade. Calcium alginate fiber (ALF) was supplied by Kangtong Marine Fiber Co., Ltd.

\subsection{Preparation of spinning dope}

The solutions including $\kappa$-carrageenan $(9 \mathrm{wt} \%, 800 \mathrm{~mL})$ and agar $(12 \mathrm{wt} \%, 800 \mathrm{~mL})$ were prepared as follows. Measured amount of $\kappa$-carrageenan powder $(72 \mathrm{~g})$ was dispersed in sodium hydroxide solution $\left(2 \mathrm{~mol} \mathrm{~L}{ }^{-1}\right)$ by stirring at $50{ }^{\circ} \mathrm{C}$ for duration of $2 \mathrm{~h}$ to obtain homogeneous carrageenan dope. For the preparation of agar solution, $96 \mathrm{~g}$ agar powder was dissolved into dimethyl sulfoxide $(704 \mathrm{~mL})$ at $95{ }^{\circ} \mathrm{C}$ for $2 \mathrm{~h}$ using a magnetic stirrer. Thereafter, the equable solutions were degassed in a vacuum oven under reduced pressure for about $30 \mathrm{~min}$. Finally, the dopes were hermetically laid aside for $12 \mathrm{~h}$ to be applied to spinning.

\subsection{Fabrication and post-processing of spinning fibers}

The fibers were produced by coagulation method using custommade wet spinning equipment in Fig. 1, and process diagram was shown in Fig. 2(a).

The spinning dopes were extruded through a commercial spinneret with thirty orifices, and the nitrogen pressure of $0.5 \mathrm{MPa}$ was controlled by a pressure regulator in order to squeeze out the dope evenly. Barium chloride was used as a precipitator, and it was dissolved in coagulating bath (7 wt\%) and stretch bath ( $4 \mathrm{wt} \%$ ). The revolving speeds of metering pump, the first roller, and the second roller were set at $4.97 \mathrm{rpm}, 10 \mathrm{rpm}$, and $12 \mathrm{rpm}$, respectively. In the next step, spinning operation could be implemented in accordance with the literature. ${ }^{31}$ Thereafter, the as-spun fibers were orderly washed by alcohol $(50 \%, \mathrm{v} / \mathrm{v})$ and alcohol $(95 \%, v / v)$ to remove residual metal ions. Finally, the obtained fibers were dried at $60{ }^{\circ} \mathrm{C}$ to a constant weight.

\subsection{Characterization}

Limiting oxygen index (LOI) was recorded using HC-2 type instrument according to international standard ASTM D2863. The fiber sample $(3 \mathrm{~g})$ was cut into strip $(10 \times 3 \times 0.4 \mathrm{~cm})$ and placed in a combustor (Fig. 2(b)), where a mixture of oxygen and nitrogen flows in an upward direction. The volume content of the oxygen was adjusted to keep the lowest oxygen concentration that just supports sustained burning.

The cone calorimeter (ISO 5660 standard) was used to evaluate the combustion behavior of fabric. Specimens with dimensions of $10 \times 10 \times 0.4 \mathrm{~cm}$ were used in all tests. According to the ISO 5660 standard, normally applied for the study of combustion behavior in plastic specimens using the cone calorimeter, the fibers ( $10 \mathrm{~g}$ ) were cut to a dimension size of $10 \times 10 \mathrm{~cm}$ and placed over the middle of an aluminum foil of $10.2 \times 10.2 \mathrm{~cm}$ with the shiny side toward the specimen. Then the specimen was pressed into a sheet with thickness of $0.4 \mathrm{~cm}$ (Fig. 2(b)). The specimens were burned for all the experiments with horizontal orientation in air atmosphere at predetermined heat fluxes of $35 \mathrm{~kW} \mathrm{~m}{ }^{-2} \cdot{ }^{38}$

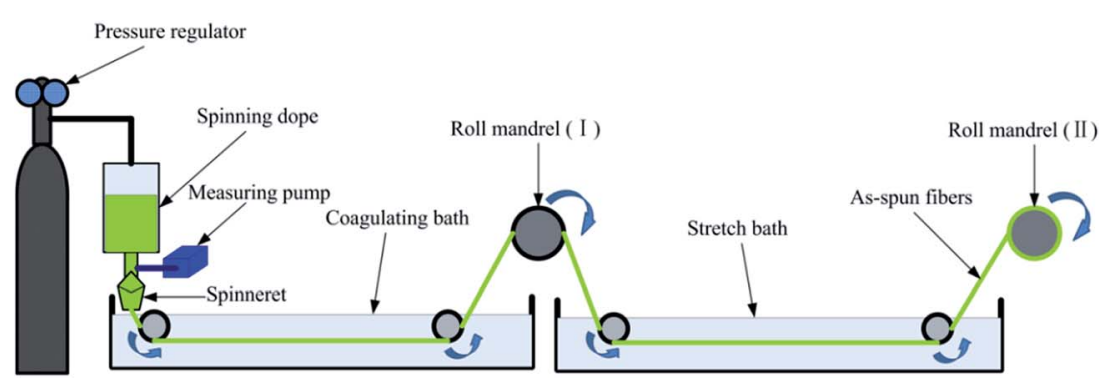

Fig. 1 Custom-made wet spinning equipment. 

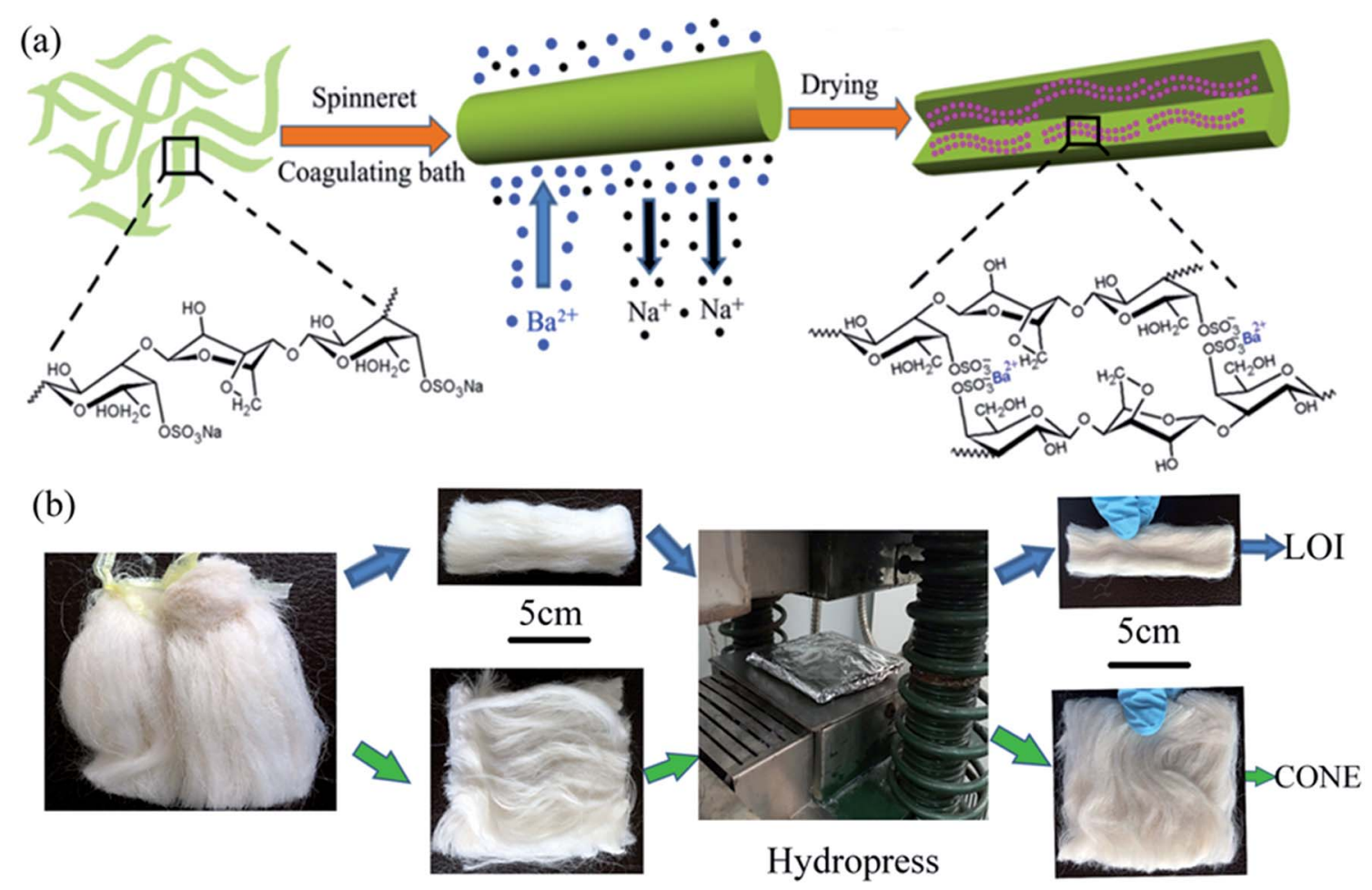

Fig. 2 (a) The scale-up process of CAF from solution to fiber. (b) The sample preparation processes of LOI and CONE.

Scanning Electron Microscope (SEM, Hitachi TM-3000, Japan) were used to record the morphology of fibers at a beam voltage of $15 \mathrm{kV}$ after gold sputtering. X-ray diffraction (XRD, DX2700, China) with an accelerating voltage of $40 \mathrm{kV}$ and a current of $30 \mathrm{~mA}$ under $\mathrm{Cu}-\mathrm{X} \alpha$ radiation was used to scan

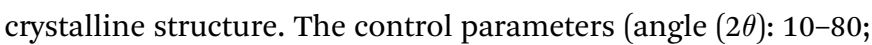
stepping angle: 0.01 ; sampling time: 0.3 ) were set appropriately.

The TG-DSC-FTIR (STA6000-Frontier, PE) measurements were conducted to record the thermal variation and functional information about the products which appeared in the process of temperature programming. About $5 \mathrm{mg}$ of the samples, under the atmosphere of nitrogen, were heated from room temperature to $900{ }^{\circ} \mathrm{C}$ at a heating rate of $20^{\circ} \mathrm{C} \mathrm{min}^{-1}$. The fast pyrolysis analyzer (Py, Frontier Lab EGA/PY-3030D, Japan) was coupled with GC-MS (QP2010Ultra, Shimadzu Corporation) systems to investigate the distribution of volatiles. The pyrolysis parameters were as follows: pyrolysis time: $0.5 \mathrm{~min}$. The GC-MS parameters were as follows: GC column (ZB-5HT): $30 \mathrm{~m} \times 0.25 \mathrm{~mm} \times 0.25 \mu \mathrm{m}$; GC temperature program: initial temperature starting at $35^{\circ} \mathrm{C}$ (kept for $3 \mathrm{~min}$ ); the heating rate: $10^{\circ} \mathrm{C} \mathrm{min}^{-1}$; carrier gas: high-purity He with the flow rate of $1.0 \mathrm{~mL} \mathrm{~min}^{-1}$; the scanning mode of ion source: full scan $(29-600 \mathrm{~m} / \mathrm{z})$. Thereafter, the compounds were determined via characterization GC-MS spectrums according to the database of NIST library.

\section{Results and discussion}

\subsection{Flame-retardant data and the influential factors}

The combustion data of ALF was acquired via LOI and CONE tests. According to previous reports, ALF possesses good flame- retardant properties. ${ }^{30}$ But, we observe that CAF presents the maximum value (52) for LOI when compared with ALF (46) and AGF (18.5). The values of LOI and CONE revealed significant differences in the fibers as shown in Table 1.

There was no TTI for CAF, keeping red state for the complete duration of test process in contrast with ALF (108 s) and AGF (4 s). The results suggested a surprising performance of CAF in regards to difficulty of combustion. In addition, the results of peak HRR, $\mathrm{THR}_{225} \mathrm{~s}, \mathrm{TSR}_{225} \mathrm{~s}$, and Mean MLR were determined to be $22 \mathrm{~kW} \mathrm{~m}{ }^{-2}, 2.9 \mathrm{MJ} \mathrm{m}^{-2}, 2.5 \mathrm{~m}^{2} \mathrm{~m}^{-2}$, and $0.03 \mathrm{~g} \mathrm{~s}^{-1}$, respectively for CAF (Table 1 and Fig. 3). The results indicated CAF had low heat release, smoke release, and mass loss in the process of combustion.

In view of the AGF, owing to the flammable characteristic and high heat release quantity, it burned more completely. And the mass was primarily lost in the form of carbon dioxide (gas state), instead of smoke due to low TSR as shown in Fig. 3(C). Therefore, AGF showed higher mean $\operatorname{MLR}\left(0.1 \mathrm{~g} \mathrm{~s}^{-1}\right)$ and lower $\mathrm{TSR}_{225 \mathrm{~s}}\left(0.8 \mathrm{~m}^{2} \mathrm{~m}^{-2}\right)$ than the other two fibers (as shown in Fig. 3(D) and (C)).

The HRR and THR trend curves of CAF shown in Fig. 3(A) and (B) are lower than that of ALF and AGF. In addition, CAF showed strong smoke suppression property in comparison with ALF (Fig. 3(C)). The phenomena indicated that there is enough time to escape in the case of sudden fires, and the flame resistance can reduce the property damage. In conclusion, the flame retardancy of CAF is superior to that of ALF and AGF.

In further investigations, we observed that pronounced changes have taken place in fiber morphology after combustion. As shown in Fig. $4\left(A_{1}\right)-\left(B_{3}\right)$, the shapes of the fibers were 
Table 1 The data of limiting oxygen index, cone calorimeter, and element content of different samples

\begin{tabular}{|c|c|c|c|}
\hline \multirow[b]{2}{*}{ Parameters } & \multicolumn{3}{|l|}{ Samples } \\
\hline & AGF & ALF & CAF \\
\hline $\mathrm{LOI}^{a}$ & 18.5 & 46 & 52 \\
\hline $\operatorname{TTI}^{b}(\mathrm{~s})$ & 4 & 108 & Red state $^{c}$ \\
\hline Peak HRR $\left(\mathrm{kW} \mathrm{m}^{-2}\right)$ & 265 & 32 & 22 \\
\hline $\mathrm{THR}_{225 \mathrm{~s}}{ }^{d}\left(\mathrm{MJ} \mathrm{m}^{-2}\right)$ & 20 & 9.6 & 2.9 \\
\hline $\operatorname{TSR}_{225 \mathrm{~s}}\left(\mathrm{~m}^{2} \mathrm{~m}^{-2}\right)$ & 0.8 & 12.1 & 2.5 \\
\hline Mean MLR $\left(\mathrm{g} \mathrm{s}^{-1}\right)$ & 0.1 & 0.05 & 0.03 \\
\hline Sulfur content $(\%)$ & 0.27 & 0 & 6.2 \\
\hline $\mathrm{Ba} / \mathrm{Ca}$ content ${ }^{e}(\%)$ & $19.4(\mathrm{Ba})$ & $36.2(\mathrm{Ca})$ & $23.3(\mathrm{Ba})$ \\
\hline
\end{tabular}

${ }^{a}$ The minimum concentration of oxygen in an oxygen/nitrogen gas stream mixture to keep combustion. ${ }^{b}$ The time needed to achieve sustained flame combustion at a particular irradiance. ${ }^{c}$ The sample keeping red state without flame. ${ }^{d}$ The total heat release in 0-225 s. ${ }^{e}$ The elements were tested after CONE.

destroyed to some degree after combustion. Fig. 4(B $\left.\mathrm{B}_{1}\right)$ shows that AGF completely lost its morphology (presenting fragmented feature). However, the residues of ALF and CAF shown in Fig. $4\left(B_{2}\right)$ and $\left(B_{3}\right)$ present relatively well morphologies which are coated with dense layer. The layer consisted of amorphous round particles or lamellar particles which closely arrange on the surface of fiber residue. Nonetheless, the surfaces of initial fibers (Fig. $4\left(\mathrm{~A}_{2}\right)$ and $\left(\mathrm{A}_{3}\right)$ ) were relatively smooth. The dense layer acted as a barrier to prevent the heat and oxygen into the fiber making the fiber produce more carbon residue. Therefore, the inside fibers turned more into black carbon residue in contrast with the surface fibers (seen from the insets of Fig. $4\left(B_{2}\right)$ and $\left(B_{3}\right)$ ).

In order to further investigate the particle composition of surface residue, X-ray diffraction was conducted under reasonable test parameters. All identified peaks in each curve could be indexed to $\mathrm{BaSO}_{4}$ (Fig. $4\left(\mathrm{C}_{1}\right)$ ), $\mathrm{CaCO}_{3}$ (Fig. $4\left(\mathrm{C}_{2}\right)$ ), and $\mathrm{BaSO}_{4}$ (Fig. $4\left(\mathrm{C}_{3}\right)$ ), respectively. The surfaces of fiber residue are covered with $\mathrm{CaCO}_{3}$ (ALF) and $\mathrm{BaSO}_{4}$ (CAF), which are proved again in Fig. 5. For AGF, the content of sulfur elements which exists in raw material was very little (Table 1 ). The residue of AGF was almost no dense layer, which was one of the reasons for its poor flame retardancy. On the contrary, the dense layer of CAF was probably the aggregation of particles of $\mathrm{BaSO}_{4}$, which could work together with carbon residues to take part in fire retardation. $^{28,29}$ Fig. $5\left(b_{1}\right)-\left(b_{4}\right)$ show that the surfaces of CAF combustion residue are covered with $\mathrm{BaSO}_{4}$ and a small amount of carbon residue. The most of carbon residues still remain in the interior of burned CAF. In addition, carrageenan molecules have certain number of sulfate ester, which could be another plausible reason behind better flame retardancy of CAF as compared to ALF or AGF. ${ }^{15,16}$
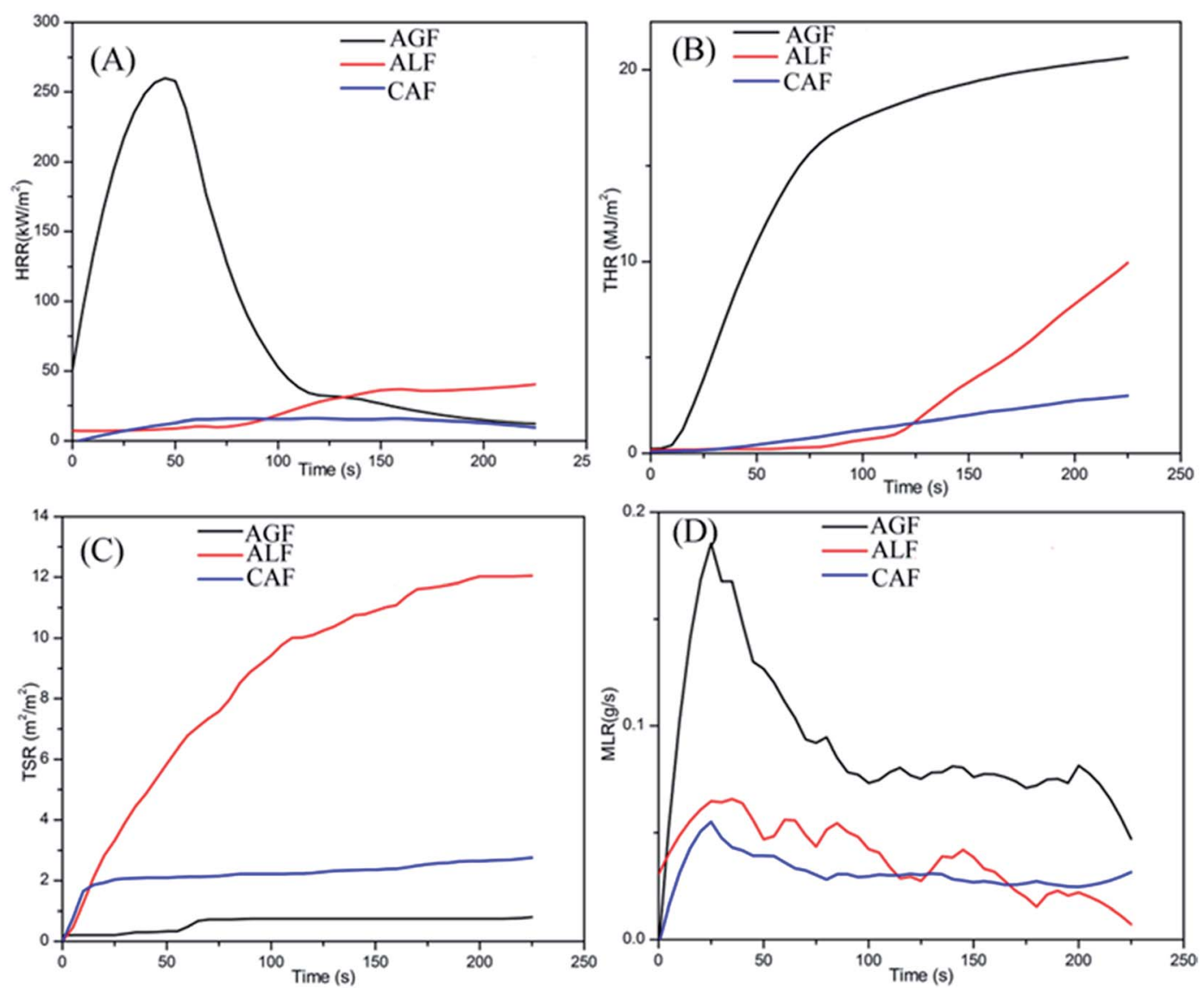

Fig. 3 The cone calorimeter data of CAF/ALF/AGF. (A) Heat release rates (HRR); (B) total heat release (THR); (C) total smoke release (TSR); (D) mass loss rate (MLR). 

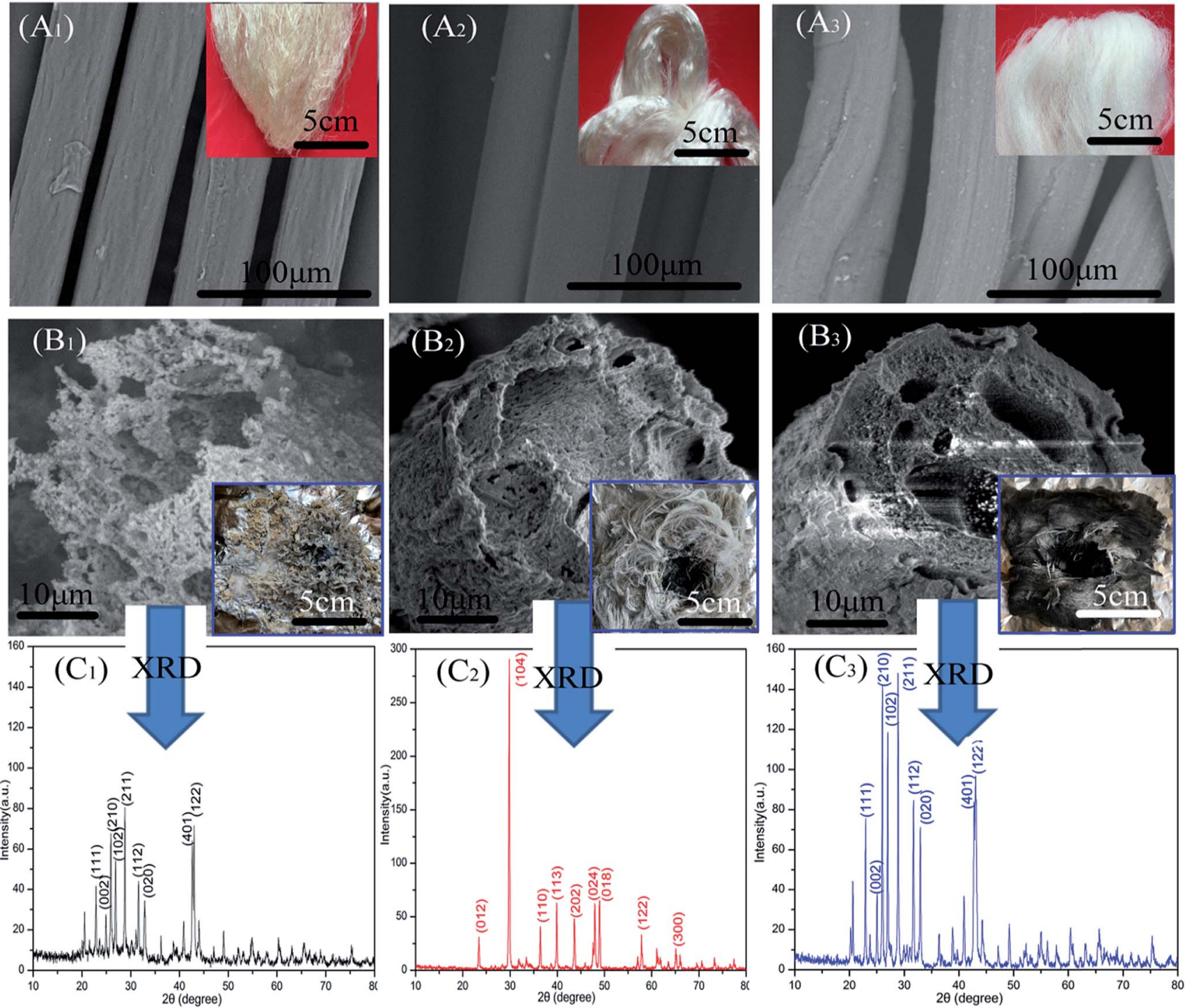

Fig. 4 SEM and optical (insets) morphology of fibers before $\left(A_{1} / A_{2} / A_{3}\right)$ and after burning $\left(B_{1} / B_{2} / B_{3}\right)$. $\left(A_{1}\right) /\left(B_{1}\right)$ Agar fiber $(A G F) ;\left(A_{2}\right) /\left(B_{2}\right)$ calcium alginate fiber $(A L F) ;\left(A_{3}\right) /\left(B_{3}\right)$ carrageenan fiber (CAF). The XRD $\left(C_{1} / C_{2} / C_{3}\right)$ of fiber residues. $\left(C_{1}\right) A G F$ residue; $\left(C_{2}\right) A L F$ residue; $\left(C_{3}\right) C A F$ residue.

\subsection{TG-DSC-FTIR analysis}

In response to the discovery of unexpected results, the flameretardant mechanism of CAF was explored with the purpose of deeper understanding of flame resistance. The results of thermal analysis data demonstrated significant differences between three kinds of fibers (Fig. 6). Fig. 6(A)-(C) indicate that all the samples exhibited initial weight loss and showed an endothermic peak on the DSC curve at about $100{ }^{\circ} \mathrm{C}$, which can be attributed to the volatilization of absorbed water of fibers. In the next step, the weight loss of AGF was up to 55\% between 200 and $400{ }^{\circ} \mathrm{C}$ which might be associated with the fracture of glycosidic bond and hexatomic ring, dehydration, and decarbonylation. ${ }^{28,39}$ Meanwhile, a great amount of heat was released and a broad exothermic peak with hysteresis appeared after $400{ }^{\circ} \mathrm{C}$, which was not conducive to the flame retardancy of AGF. As shown in Fig. 6(B), similar to CAF (Fig. 6(C)), there were four stages of mass loss in the process of temperature-rise, which can be seen from the curve of DTG. The maximum weight loss rates of AGF, ALF and CAF, located in the second stage of the DTG curves, were about $12 \%$ per min, $11.5 \%$ per min and $30 \%$ per min, respectively, while the corresponding weight losses seen from the curves of TG were about 55\%, 35\% and $13 \%$, respectively. These phenomena can be attributed to the better thermostability of CAF. Fig. 6(C) shows that CAF exhibited a large endothermic peak in the fourth stage when the temperature rose to $800{ }^{\circ} \mathrm{C}$, and the weight loss was about $12 \%$ in this stage which can be attributed to the further decomposition of carbon residue. These results suggested that CAF exhibited superior high-temperature resistance due to the endothermic effect under high temperature. Also, as observed from the curves of TG, when the temperature was up to $700{ }^{\circ} \mathrm{C}$, the rest mass of the three kinds of fiber was 25\% (AGF), 35\% (ALF), and 45\% (CAF), respectively. These results indicated that 

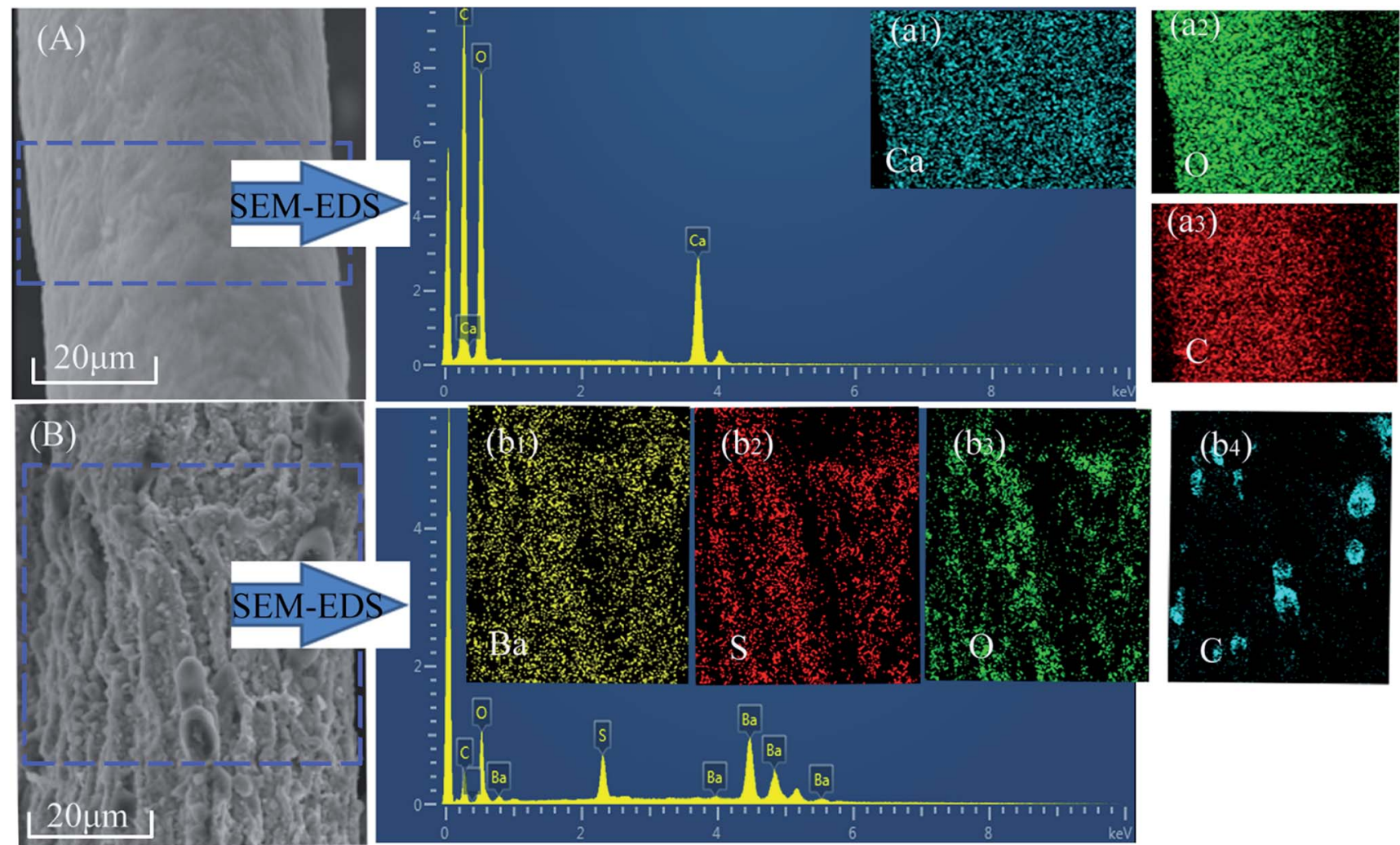

Fig. 5 SEM-EDS test of the surfaces of ALF (A) and CAF (B) combustion residue.

many carbonized products were formed for CAF, suggesting another plausible reason behind flame-retardant performance.

FTIR technique was utilized for characterization of gaseous pyrolysis products of polymers, which exhibits different infrared characteristic peaks at different positions. Overall pyrolysis products can be easily found from the three- dimensional diagram of TG-FTIR. As observed in Fig. 6(a) and (b), AGF and ALF produced large amounts of pyrolysis products at the beginning time. In contrast, CAF began to produce a significant amount of pyrolysis gas at around $2000 \mathrm{~s}$ (Fig. 6(c)), and there were distinct absorption peaks at $2340 \mathrm{~cm}^{-1}$, $2200 \mathrm{~cm}^{-1}, 1700 \mathrm{~cm}^{-1}$ and $1100 \mathrm{~cm}^{-1}$. Additionally, the
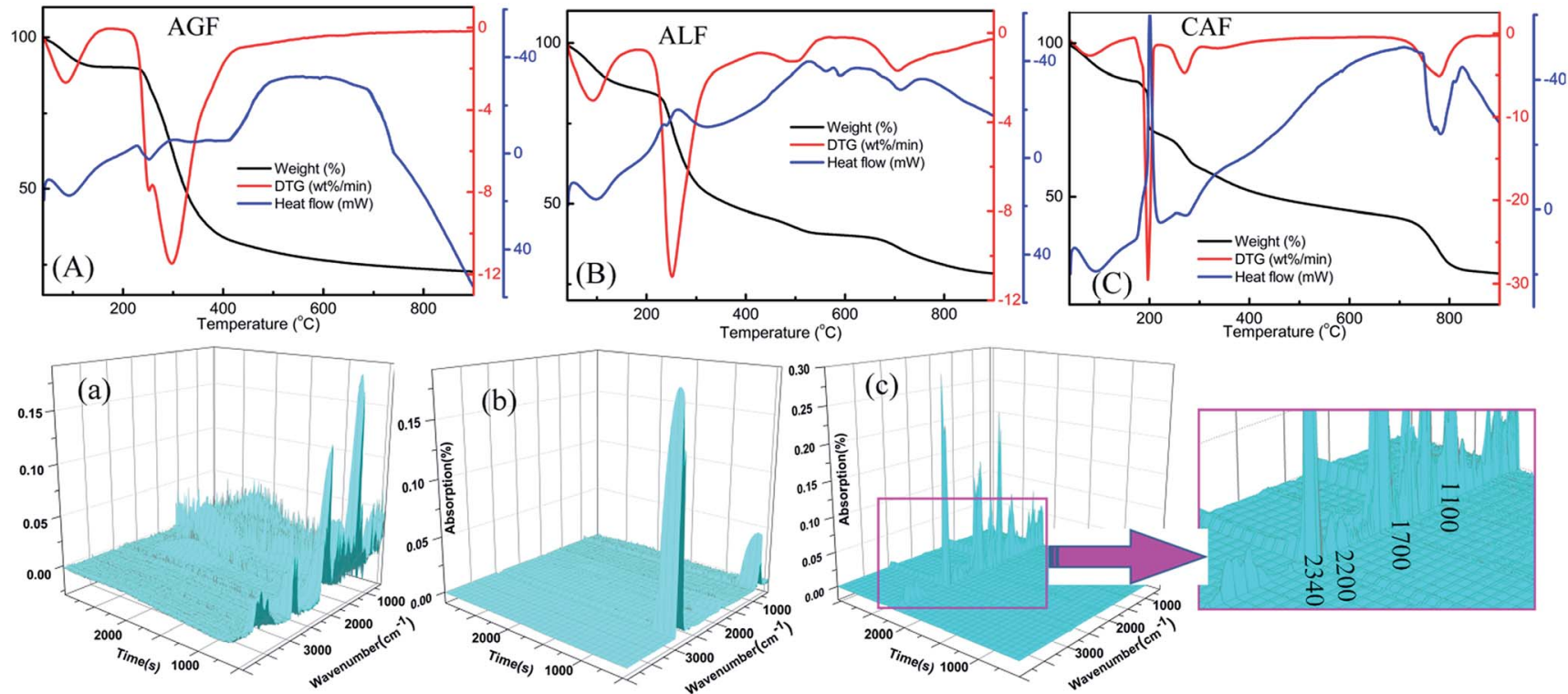

(b)
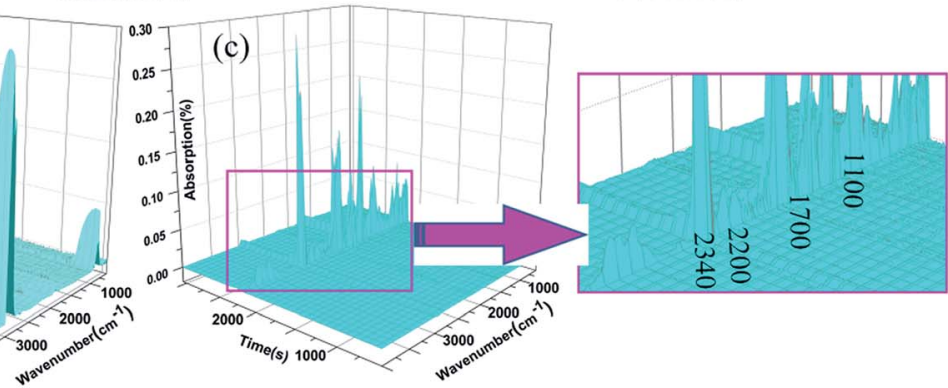

Fig. 6 TG-DSC thermograms of AGF (A), ALF (B), and CAF (C) and three-dimensional diagram of TG-FTIR of AGF (a), ALF (b) and CAF (c). 
pyrolysis products of CAF were less than that of AGF, as shown in Fig. 6(a) and (c). The results also showed that CAF had better high temperature resistance than AGF or ALF.

In the above context, infrared spectra of special groups which were recorded near the temperatures of major weight loss rate were compared in Fig. 7. For example, AGF: 100, 200, 300, and $500{ }^{\circ} \mathrm{C}$ were typical temperature according to Fig. 6(A), and the temperature of the maximum weight loss rate was $300{ }^{\circ} \mathrm{C}$; ALF: $100,250,550$, and $750{ }^{\circ} \mathrm{C}$ were typical temperature according to Fig. 6(B), and the temperature of the maximum weight loss rate was $250{ }^{\circ} \mathrm{C}$; CAF: $100,210,280,725$, and $780{ }^{\circ} \mathrm{C}$ were typical temperature according to Fig. 6(C), and the temperatures of the maximum weight loss rate was 210 and $780{ }^{\circ} \mathrm{C}$.

As shown in Fig. 7, the maximum absorption intensity of three kinds of fiber was recorded around $300{ }^{\circ} \mathrm{C}$ (AGF), $250{ }^{\circ} \mathrm{C}$ (ALF), and $725^{\circ} \mathrm{C}(\mathrm{CAF})$, respectively. The broad band at 3500$3800 \mathrm{~cm}^{-1}$ was related to $\mathrm{O}-\mathrm{H}$ stretching vibration of pyrolysis gas; the peak at $2340 \mathrm{~cm}^{-1}$ was attributed to $\mathrm{CO}_{2}$, and the peak around $1700 \mathrm{~cm}^{-1}$ was assigned to $\mathrm{C}=\mathrm{O}$ stretching vibration which was rather stronger in Fig. 7 (a) $\left(300^{\circ} \mathrm{C}\right)$. The peak at 2200 $\mathrm{cm}^{-1}$ was determined to $\mathrm{C} \equiv \mathrm{C}$ stretching vibration or gaseous phase $\mathrm{CO}$ which was relatively stronger in Fig. $7(\mathrm{c})\left(725^{\circ} \mathrm{C}\right)$. The bands at 1000-1300 and 1620-1680 $\mathrm{cm}^{-1}$ which was overlapped with the absorbance peak of $\mathrm{C}=\mathrm{O}$ were identified as $\mathrm{C}-\mathrm{O}$ and $\mathrm{C}=\mathrm{C}$, respectively, and they were distinct in Fig. 7(a). Based on the results in Fig. 7(a)-(c), we can conclude that with the rise in temperature, the concentration of pyrolysis gas first increases and then decreases with further rise in temperature. Also, AGF generated many complex and abundant pyrolysis products at $300{ }^{\circ} \mathrm{C}$ which was in accordance with Fig. 6(a). CAF released less pyrolysis gas at relatively low temperature range, and significant amount of pyrolysis gas was produced in the high temperature range (Fig. 6(c) and 7(c)). The results further demonstrated that CAF had better flame retardancy than the other two fibers. Moreover, high concentration of carbon dioxide in pyrolysis gas further promoted the flame-retardant ability for CAF.

FTIR spectra of fiber residues are presented in Fig. 7(d). Since CAF produces bulk of carbon residues which can form conjugate systems, the system promoted the band at 1620-1680 $\mathrm{cm}^{-1}$ to move around $1600 \mathrm{~cm}^{-1}$ and the conjugate system further improved the stability of carbon residues. ${ }^{\mathbf{4 0 - 4 3}}$ Moreover, it was noted that there was distinct absorption band at 1040$1200 \mathrm{~cm}^{-1}$ which was attributed to $\mathrm{S}=\mathrm{O}$ stretching vibration. This group can exert an important influence on pyrolysis mechanism of carrageenan. ${ }^{\mathbf{1 5}, 16}$ Also, $\mathrm{S}=\mathrm{O}$ intensity became
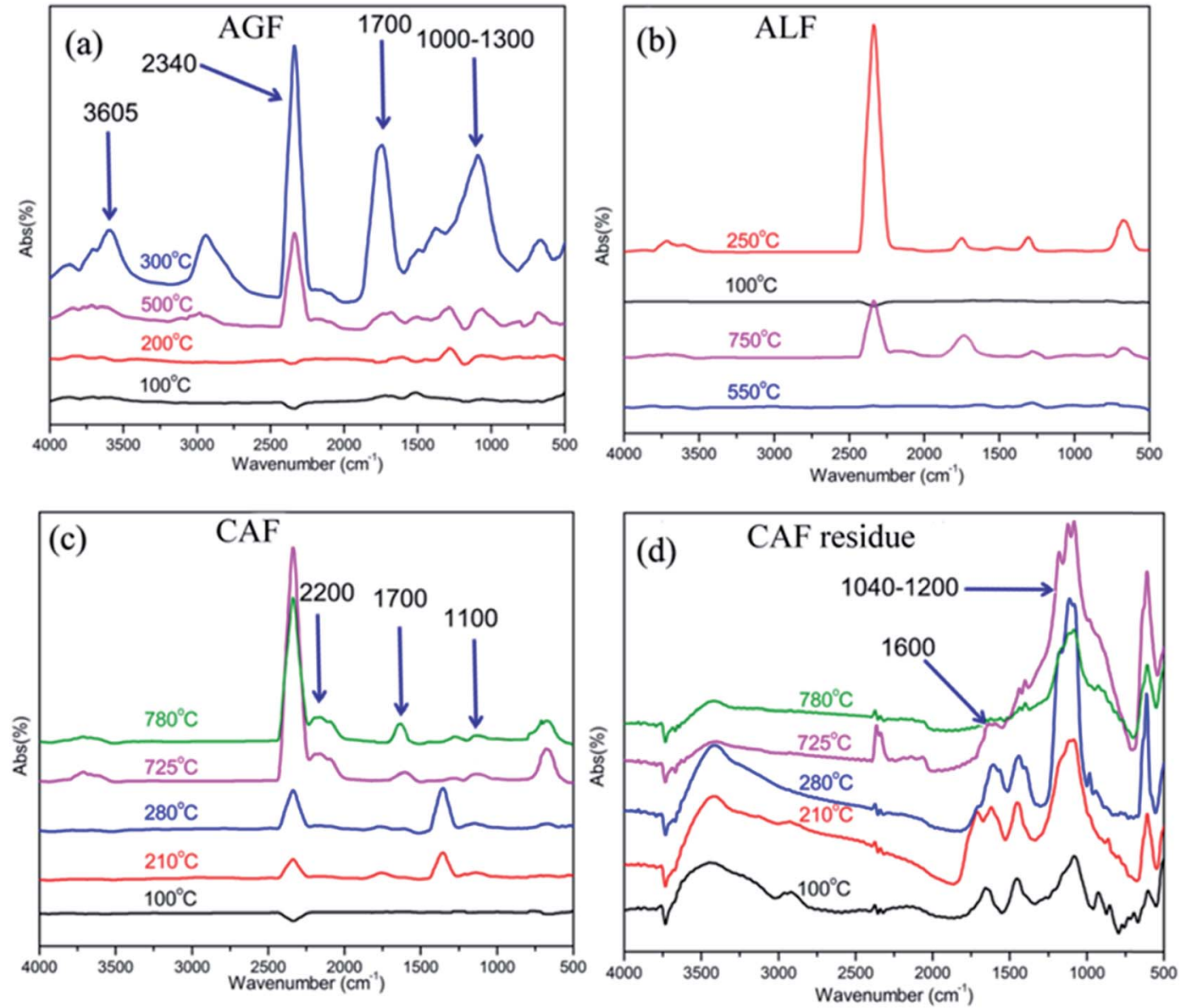

Fig. 7 FTIR spectra of thermal degradation products of fibers in different temperatures (programmed temperature in accordance with TG in Fig. 6). (a) AGF; (b) ALF; (c) CAF; (d) CAF residue. 
stronger with an increase in temperature. The groups of $\mathrm{S}=\mathrm{O}$ primarily remain in residue rather than releasing in pyrolysis gas, which fully embodies the principle of environmental protection.

\subsection{Py-GC-MS analysis}

In order to investigate the primary components of gaseous products in the pyrolysis process of CAF, pyrolysis gas chromatography and mass spectrometry (Py-GC-MS) analysis were conducted at $250{ }^{\circ} \mathrm{C}$ and $750{ }^{\circ} \mathrm{C}$ (near the higher value of weightlessness) which were selected according to the curve of TG. AGF and ALF which exhibited relatively poor flame retardancy in comparison with CAF were not analyzed in Py-GC-MS test. In comparison with mass spectra fragmentation pattern with NIST library and published data, the highest similarity of compound identification was obtained. The gas chromatogram demonstrating the peaks of different retention time is displayed in Fig. 8, and the major characteristic compounds are summarized in Table 2.

Fig. 8(a) shows that when the pyrolysis temperature was $250{ }^{\circ} \mathrm{C}$, there were fewer peaks in the gas chromatogram as compared to $750{ }^{\circ} \mathrm{C}$ (more than 20 peaks). These results indicated that CAF still had good stability at $250{ }^{\circ} \mathrm{C}$. As shown in Table 2, when the temperature was set at $250{ }^{\circ} \mathrm{C}$, the primary component of pyrolysis gas was $\mathrm{CO}_{2}$ (about $72.06 \%$ ) which played an important role in preventing combustion. The primary pyrolytic products (shown in Fig. 8 and Table 2) were as follows: (a): (1) carbon dioxide, (2) butan-2-ol, (3) cyclohexadeca1,9-diene, (4) 4-butyl-cyclohexene, (5) 4-isobutyl-cyclohexene; (b): (1) 2-oxo-3-sulfo-propionic acid, (2) pent-3-yn-1-ol, (3) acetic acid 3-methyl-but-3-enyl ester, (4) cyclopenta-1,3-diene, (5) 2methyl-furan, (6) hexa-1,3-dien-5-yne, (7) 2,5-dimethyl-furan, (8) pentan-2-one, (9) 2,4-dimethyl-furan, (10) spiro[2.4]hepta-4,6diene, (11) 1-furan-2-yl-ethanone, (12) 3-methylene-1-vinylcyclopentene. These compounds could be primarily divided into furan, ketone, unsaturated hydrocarbon, hydroxyl compound, acids, and they contain the functional information of $\mathrm{C}=\mathrm{C}, \mathrm{C}=\mathrm{O}, \mathrm{C} \equiv \mathrm{C}$ and $\mathrm{S}=\mathrm{O}$ which were in accordance with the data of TG-FTIR in Fig. 6(c) and 7(c). The mass of these compounds was smaller than that of carbon residue which was incumbent on the surface of the fiber to stop the unceasing production of pyrolysis gas. As shown in Table 2, at $750{ }^{\circ} \mathrm{C}, 2$ oxo-3-sulfo-propionic acid was the primary products along with carbon dioxide whose total yield was $23.92 \%$. The results indicate that sulfate group might have a great influence on flameretardant mechanism because of easy formation of sulfonyl free radical (SFR) under heating conditions. SFR was produced under the heating conditions, and it was apt to combine with hydroxyl radical (HR) generating sulfo group (Scheme 1c). The reduction of HR was in favor of fire resistance. In the next step,

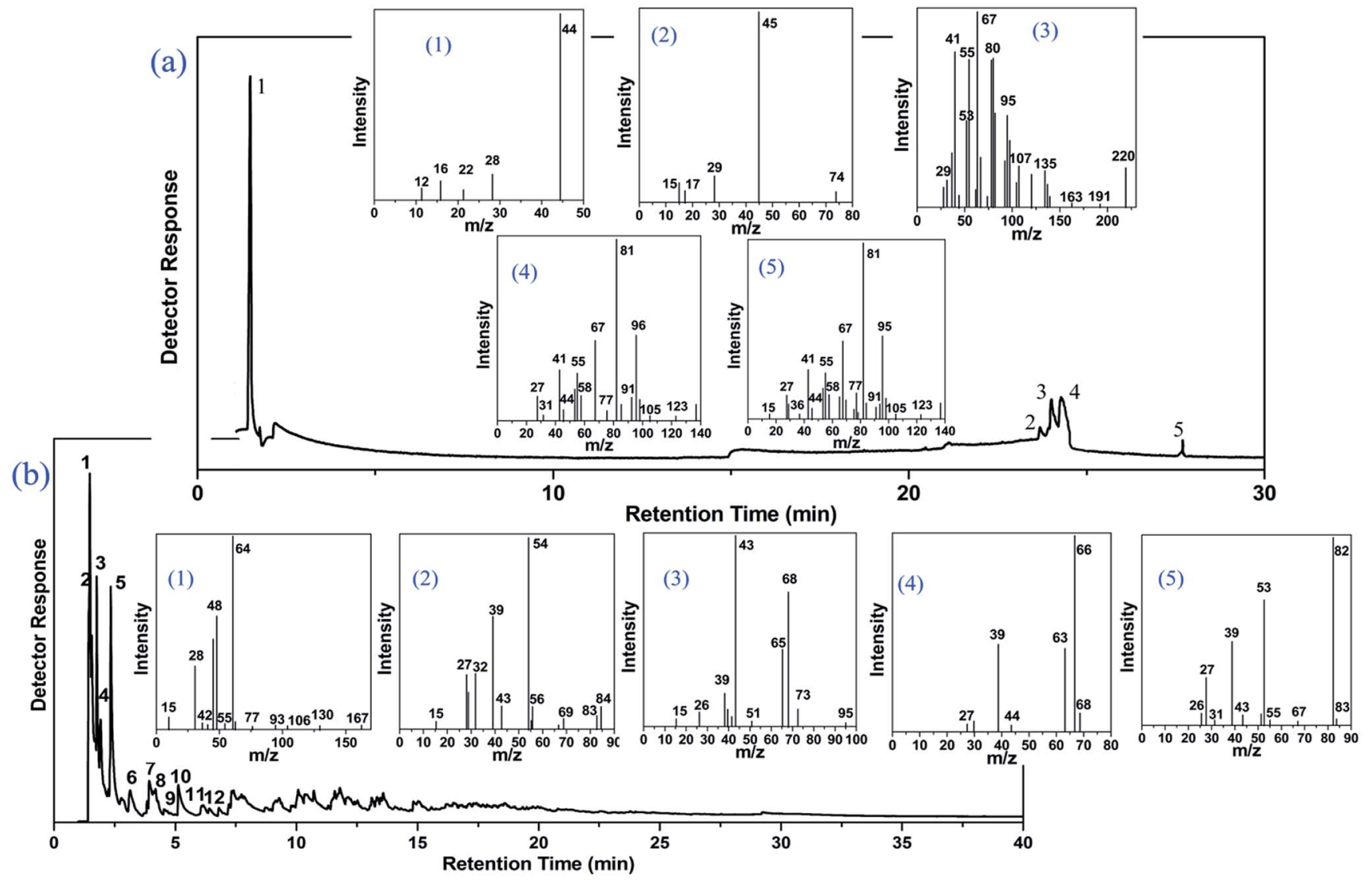

Fig. 8 Py-GC-MS spectra of CAF under the different pyrolysis temperatures. (a) $250^{\circ} \mathrm{C}$; (b) $750^{\circ} \mathrm{C}$ (the pyrolysis products of $6-12$ are not listed in MS spectra). 
Table 2 Py-GC-MS main analysis results of CAF at 250 and $750^{\circ} \mathrm{C}$

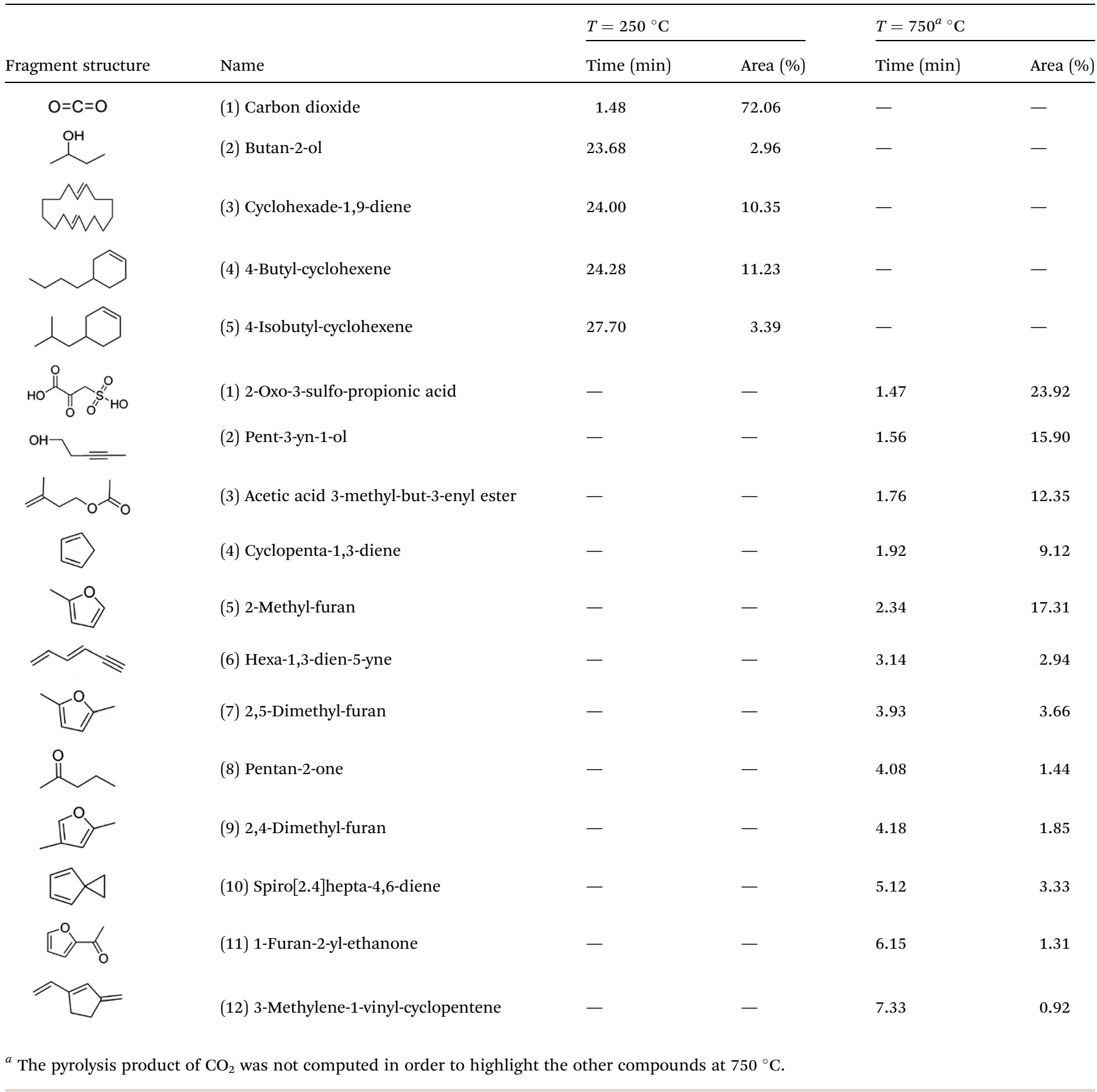

sulfo group combined with carbon monoxide and hydrogen free radical (HFR) reproducing SFR along with the production of $\mathrm{CO}_{2}$ and $\mathrm{H}_{2} \mathrm{O} .{ }^{44-49}$ The pyrolysis mechanism is presented in Scheme 1(a)-(d). Moreover, sulfate groups can combine with barium ions generating a layer of barium sulfate (seen from Fig. $4\left(\mathrm{~B}_{3}\right)$ and $\left(\mathrm{C}_{3}\right)$ and $\left.5(\mathrm{~B})\right)$, which can prevent the exchange of matter and energy and stop them in and out of the fiber.

In accordance with the TG-DSC-FTIR and Py-GC-MS analyses in inert gas atmosphere, pyrolysis mechanisms of CAF are summarized in Scheme 1. It shows the mechanism of carbon residue (condensed phase mechanism), SFR (the gas phase mechanism), barium sulfate (condensed phase mechanism), water vapor (the gas phase mechanism), and carbon dioxide (the gas phase mechanism) which are marked by multigonal star pattern in Scheme 1, and three primary cracking paths are listed in Scheme 1(b). The intermediate products were further degraded under the condition of heating through bond breaking, ring opening, dehydration, desulfuration, ring formation and so on. Then, some of the molecules and fracture fragments, seen from Scheme 1(c), were produced and detected. They have great guiding significance for flame-retardant mechanism of CAF in the air atmosphere. 
(a)

(b)

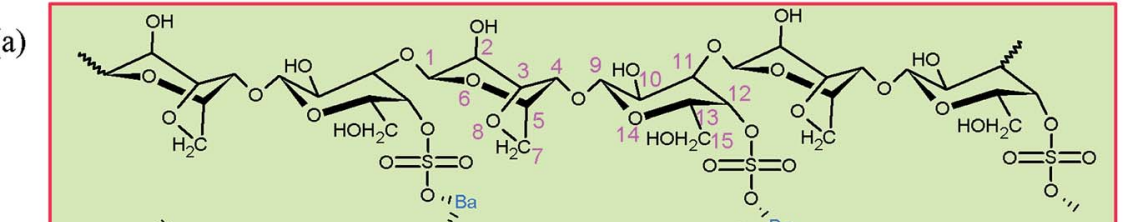

(b)
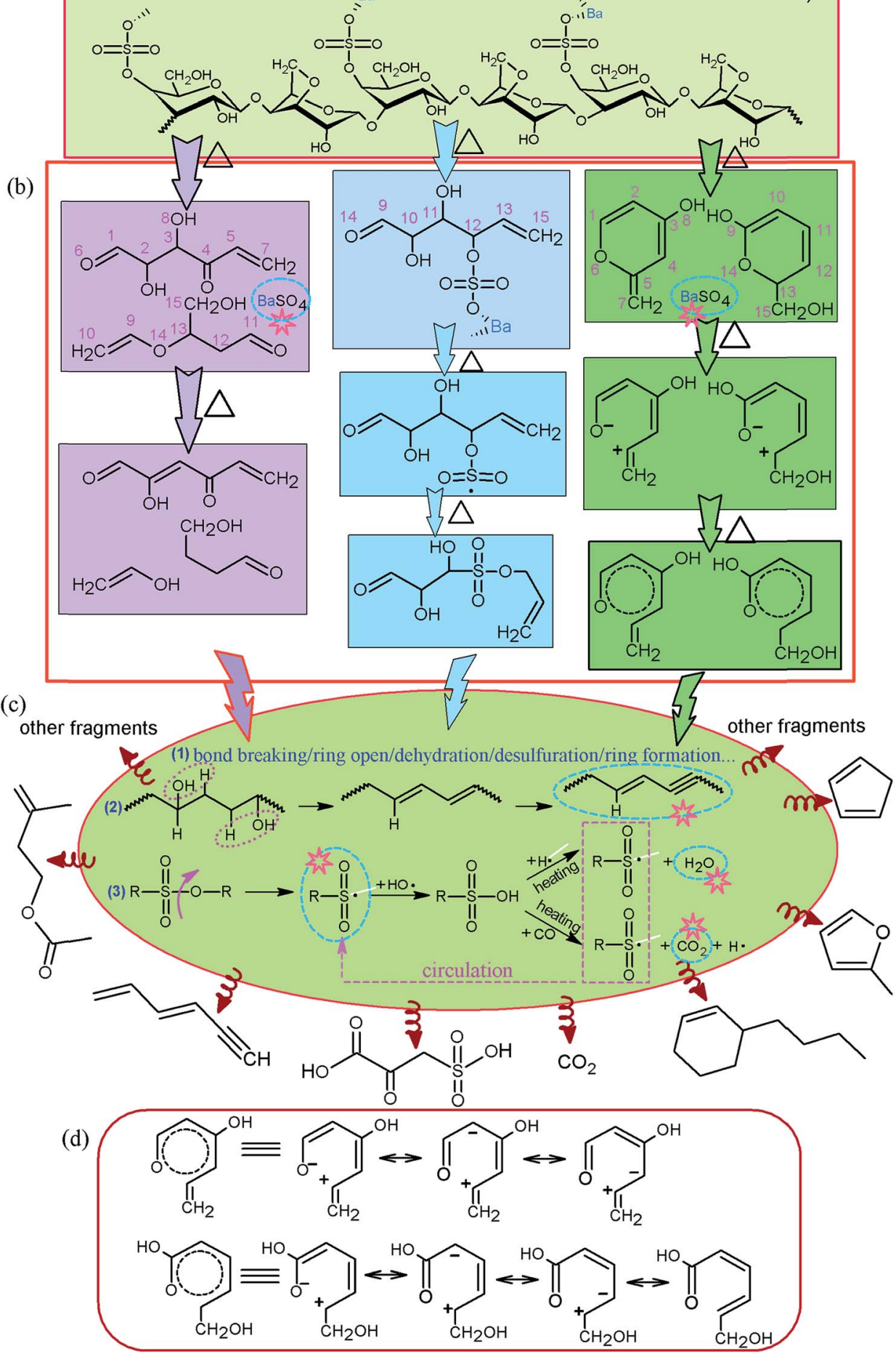

Scheme 1 Schematic representation of proposed pyrolysis mechanisms of CAF. (a) Polymeric macromolecule of CAF. (b) The main cracking paths under the condition of heating. (c) Pyrolysis mechanisms and pyrolysis products. (d) The explanation of some cracking intermediates. 


\section{Conclusion}

The data of LOI (52) and CONE showed that CAF exerts difficultflammability and low heat yield in contrast with AGF or ALF. The results of TG showed that when the temperature was up to $700{ }^{\circ} \mathrm{C}$, the rest masses of the three kinds of fiber were $25 \%$ (AGF), 35\% (ALF), and 45\% (CAF), respectively. In comparison with the previous reports on flame-retardant alginate fiber, $\mathrm{CAF}$ demonstrated much stronger flame retardancy, most likely due to the distinct flame-retardant mechanisms such as SFR, carbon residue, barium sulfate, and carbon dioxide. SFR can reduce the concentration of HFR to terminate the combustion. Moreover, unburned fiber was wrapped by carbon residue and barium sulfate which were capable of preventing heat going into the fiber. In general, CAF, a new type of flame-retardant material, is showing a great utilization potential. Next, we will try to add other metal ions and flame retardants to further improve the flame retardancy of CAF. In particular, the flame-retardant mechanism of CAF should be further explored.

\section{Acknowledgements}

This work is supported by the National Natural Science Foundation of China (50803030), the Postdoctoral Science Special Foundation of China (201104581), the Postdoctoral Science Foundation of China (20100471495), the Program for Taishan Scholar of Shandong Province of China, and the Program for Changjiang Scholars and Innovative Research Team in University of China (No. IRT0970). The authors are also thankful to the International Cooperation Program for Excellent Lectures of 2012 by Shandong Provincial Education Department of China and the Special Fund for Self-directed Innovation of Shandong Province of China (No. 2013CXB80201).

\section{Notes and references}

1 S. Rudhziah, et al., Potential of blend of kappa-carrageenan and cellulose derivatives for green polymer electrolyte application, Ind. Crops Prod., 2015, 72, 133-141.

$2 \mathrm{~K}$. Ako, Influence of elasticity on the syneresis properties of kappa-carrageenan gels, Carbohydr. Polym., 2015, 115, 408414.

3 L. Piculell, et al., t-Carrageenan Is Excluded from the Chiral Nematic Phase of $\kappa$-Carrageenan, Macromolecules, 1998, 31, 5152-5154.

4 F. M. Almutairi, et al., An analytical ultracentrifugation based study on the conformation of lambda carrageenan in aqueous solution, Carbohydr. Polym., 2013, 97(1), 203-209.

5 F. Cuppo, et al., Association of $\kappa$-Carrageenan Induced by $\mathrm{Cs}^{+}$ Ions in Iodide Aqueous, Macromolecules, 2002, 35, 539-547.

6 G. Michel, et al., Bioconversion of red seaweed galactans: a focus on bacterial agarases and carrageenases, Appl. Microbiol. Biotechnol., 2006, 71(1), 23-33.

7 S. Liu, et al., Rheological Properties and Scaling Laws of $\kappa$ Carrageenan in Aqueous Solution, Macromolecules, 2015, 48(20), 7649-7657.
8 M. Raman, et al., $\kappa-$ Carrageenan from marine red algae, Kappaphycus alvarezii - a functional food to prevent colon carcinogenesis, J. Funct. Foods, 2015, 15, 354-364.

9 M. Darder, et al., Bio-Nanocomposites Based on Layered Double Hydroxides, Chem. Mater., 2005, 17, 1969-1977.

10 L. V. Abad, et al., Emerging applications of radiationmodified carrageenans, Nucl. Instrum. Methods Phys. Res., Sect. B, 2014, 336, 167-172.

11 V. A. Cosenza, et al., Partial and total C-6 oxidation of gelling carrageenans. Modulation of the antiviral activity with the anionic character, Carbohydr. Polym., 2015, 128, 199-206.

12 P. Desftischer, et al., Chemical structure and antiviral activity of carrageenans from Meristiella gelidium against herpes simplex and dengue virus, Carbohydr. Polym., 2006, 63(4), 459-465.

13 M. E. R. Duarte, et al., The structure of the agaran sulfate from Acanthophora spicifera (Rhodomelaceae, Ceramiales) and its antiviral activity. Relation between structure and antiviral activity in agarans, Carbohydr. Res., 2004, 339(2), 335-347.

14 A. C. Estrada, et al., Photothermally enhanced drug release by $\kappa$-carrageenan hydrogels reinforced with multi-walled carbon nanotubes, RSC Adv., 2013, 3, 10828-10836.

$15 \mathrm{Z}$. Li, et al., Flame retardancy, thermal and mechanical properties of sulfonate-containing polyhedral oligomeric silsesquioxane (S-POSS)/polycarbonate composites, Polym. Degrad. Stab., 2015, 116, 81-87.

$16 \mathrm{~S}$. Liu, et al., Study on flame-retardant mechanism of polycarbonate containing sulfonate-silsesquioxane-fluoro retardants by TGA and FTIR, Polym. Degrad. Stab., 2006, 91(8), 1808-1814.

17 G. Abbasi, et al., Product screening for sources of halogenated flame retardants in Canadian house and office dust, Sci. Total Environ., 2015, 545, 299-307.

18 A. Abou-Okeil, et al., Flame retardant cotton fabrics treated with organophosphorus polymer, Carbohydr. Polym., 2013, 92(2), 2293-2298.

19 M. M. El-Hady, et al., Flame retardancy and UV protection of cotton based fabrics using nano $\mathrm{ZnO}$ and polycarboxylic acids, Carbohydr. Polym., 2013, 92(1), 400-406.

20 C. K. Poon, et al., Effects of $\mathrm{TiO}_{2}$ and curing temperatures on flame retardant finishing of cotton, Carbohydr. Polym., 2015, 121, 457-467.

21 L. Qian, et al., Thermal degradation behavior of the compound containing phosphaphenanthrene and phosphazene groups and its flame retardant mechanism on epoxy resin, Polymer, 2011, 52(24), 5486-5493.

22 Q. Wang, et al., Synthesis of Flame-Retardant Polypropylene/ LDH-Borate Nanocomposites, Macromolecules, 2013, 46(15), 6145-6150.

$23 \mathrm{~K}$. Xie, et al., Flame retardant finishing of cotton fabric based on synergistic compounds containing boron and nitrogen, Carbohydr. Polym., 2013, 98(1), 706-710.

24 S. Yang, et al., Synthesis of a phosphorus/nitrogencontaining compound based on maleimide and cyclotriphosphazene and its flame-retardant mechanism on epoxy resin, Polym. Degrad. Stab., 2016, 126, 9-16. 
25 J. Zhang, Q. Ji, F. Wang, L. Tan and Y. Xia, Effects of divalent metal ions on the flame retardancy and pyrolysis products of alginate fibres, Polym. Degrad. Stab., 2012, 97(6), 1034-1040.

26 J. Zhang, Q. Ji, P. Zhang and Y. Xia, Thermal stability and flame-retardancy mechanism of poly(ethylene terephthalate)/ boehmite nanocomposites, Polym. Degrad. Stab., 2010, 95(7), 1211-1218.

27 K. Zhang, L. Zong, Y. Tan, Q. Ji, W. Yun, R. Shi and Y. Xia, Improve the flame retardancy of cellulose fibers by grafting zinc ion, Carbohydr. Polym., 2016, 136, 121-127.

28 Y. Liu, et al., Bio-based barium alginate film: preparation, flame retardancy and thermal degradation behavior, Carbohydr. Polym., 2016, 139, 106-114.

29 Y. Liu, et al., Effect of reactive time on flame retardancy and thermal degradation behavior of bio-based zinc alginate film, Polym. Degrad. Stab., 2016, 127, 20-31.

$30 \mathrm{~J}$. Zhang, et al., Pyrolysis products and thermal degradation mechanism of intrinsically flame-retardant calcium alginate fibre, Polym. Degrad. Stab., 2011, 96(5), 936-942.

$31 \mathrm{~W}$. Zhang, et al., Effect of epichlorohydrin on the wet spinning of carrageenan fibers under optimal parameter conditions, Carbohydr. Polym., 2016, 150, 232-240.

$32 \mathrm{P}$. Croguennoc, et al., Characterization of Semidilute $\kappa$ Carrageenan Solutions, Macromolecules, 2000, 33, 7471-7474.

33 L. Kong, Fabrication of $\kappa$-carrageenan fibers by wet spinning: addition of ı-carrageenan, Food Hydrocolloids, 2013, 30(1), 302-306.

34 M. Matsuo, et al., Gelation mechanism of agarose and $\kappa-$ carrageenan solutions estimated in terms of concentration fluctuation, Polymer, 2002, 43, 5299-5309.

35 B. K. Patel, et al., Impact of urea on the three-dimensional structure, viscoelastic and thermal behavior of iotacarrageenan, Carbohydr. Polym., 2013, 92(2), 1873-1879.

36 A. Rosas-Durazo, et al., Gelation processes in the nonstoichiometric polylectrolyte-surfactant complex between $\kappa$-carrageenan and dodecyltrimethylammonium chloride in KCl, Soft Matter, 2011, 7(5), 2103-2112.

37 B. Bhowmick, et al., Effect of carrageenan and potassium chloride on an in situ gelling ophthalmic drug delivery system based on methylcellulose, RSC Adv., 2015, 5, 6038660391.

$38 \mathrm{~J}$. Tata, et al., Optimization of the procedure to burn textile fabrics by cone calorimeter: part I. Combustion behavior of polyester, Fire Mater., 2011, 35(6), 397-409.
39 H. B. Chen, et al., Low flammability, foam-like materials based on ammonium alginate and sodium montmorillonite clay, Polymer, 2012, 53(25), 5825-5831.

$40 \mathrm{~F}$. Chen, et al., Synthesis, optical properties and stability of two $\sigma-\pi$ conjugated polycarbosilanes with silylene-1,2diphenyleneethylene-silylene and silylene-terephthalyidenesilylene backbones via Grignard reactions, Synth. Met., 2016, 221, 253-260.

$41 \mathrm{H}$. Gaspar, et al., Increase in thermo-oxidation stability of conjugated polymers at high temperatures, Polym. Test., 2014, 34, 183-191.

42 S. B. Jadhav, et al., Screening of polysaccharides for preparation of alpha-amylase conjugate to enhance stability and storage life, Carbohydr. Polym., 2013, 92(2), 1724-1729.

43 G. Li, et al., Hydroxyethyl starch conjugates for improving the stability, pharmacokinetic behavior and antitumor activity of 10-hydroxy camptothecin, Int. J. Pharm., 2014, 471(1-2), 234-244.

$44 \mathrm{Y}$. Chen, et al., A novel flame retardant containing phosphorus, nitrogen, and sulfur,, J. Therm. Anal. Calorim., 2013, 115(2), 1639-1649.

45 K. Dai, et al., Enhanced Properties of the Incorporation of a Novel Reactive Phosphorus- and Sulfur-Containing Flame-Retardant Monomer into Unsaturated Polyester Resin, Ind. Eng. Chem. Res., 2012, 51(49), 15918-15926.

46 S. A. Everett, et al., Scavenging of nitrogen dioxide, thiyl, and sulfonyl free radicals by the nutritional antioxidant betacarotene, J. Biol. Chem., 1996, 271(8), 3988-3994.

47 M. S. Karaivanona, et al., Non-halogen-containing flameretardant ethylene-propylene copolymer compositions for cable insulation with nitrogen-and sulfur-containing fire retardants, J. Appl. Polym. Sci., 1997, 63(5), 581-588.

$48 \mathrm{~V}$. Percec, et al., Scope and limitations of functional sulfonyl chlorides as initiators for metal-catalyzed "living" radical polymerization of styrene and methacrylates, Macromolecules, 1997, 30(25), 8526-8528.

49 A. Ryokawa, et al., Synthetic use of 1,1,2,2tetraphenyldisilane for the preparation of biaryls through the intramolecular free radical ipso-substitution of $\mathrm{N}$-(2bromoaryl) arenesulfonamides, Tetrahedron, 2001, 57(28), 5915-5921. 